\title{
Optoacoustic Imaging of Naphthalocyanine: Potential for Contrast Enhancement and Therapy Monitoring
}

\author{
Nicolas Bézière and Vasilis Ntziachristos \\ Institute for Biological and Medical Imaging, Helmholtz Zentrum München and Technische Universität München, Neuherberg, \\ Germany
}

\begin{abstract}
We investigated in vitro and in vivo the optoacoustic responses of silicon 2,3-naphthalocyanine bi(trihexylsilyloxide) (SiNc), considered herein as a reporter molecule for optoacoustic imaging, elucidating its efficiency for optoacoustic (photoacoustic) signal generation and examining the in vivo performance achieved. Methods: SiNc solutions were prepared using Cremophor EL in water and evaluated for light-absorbing and optoacoustic contrast-generating properties. Photostability and singlet oxygen generation were investigated under pulsed laser illumination and validated using photoabsorbance. HT-29 mouse tumor models were used to assess the biodistribution of the compound and its performance as an optoacoustic contrast agent in vivo. Results: SiNc was found to generate superior optoacoustic signals compared with the commonly used indocyanine green. Multispectral optoacoustic tomography of mouse tumors efficiently resolved the biodistribution of $\mathrm{SiNc}$ and the underlying perfusion parameters in vivo. In addition, we demonstrate how lighttriggered SiNc reactions with molecular oxygen can be potentially sensed and discuss the relation of these measurements to the biochemical process involved in photothermal treatment. Conclusion: SiNc appears to be a promising family of contrast agent for optoacoustic imaging. Further development possibilities promise to expand its use in purely contrast generation settings, as well as its photodynamic therapy application.
\end{abstract}

Key Words: naphthalocyanine; photodynamic therapy; optoacoustic; photoacoustic; imaging

J Nucl Med 2015; 56:323-328

DOI: 10.2967/jnumed.114.147157

\section{$\mathbf{P}$}

hthalocyanines are strongly colored macrocyclic compounds that represent a class of artificial pigments. Structurally related to porphyrin, they share the tetradentate property and can complex metals. Interestingly, metal complexation and substitution on the benzene ring of the isoindole allow a red shift of the absorbance spectra (1). The family of molecules of interest herein, naphthalocyanine, was built using benzoisoindole instead of isoindole blocks and exhibits a sharp and intense absorbance band in the near-infrared region of the spectra (700-900 nm). After light ab-

Received Aug. 20, 2014; revision accepted Nov. 7, 2014.

For correspondence or reprints contact: Nicolas Bézière, Institute for Biological and Medical Imaging, Helmholtz Zentrum München and Technische Universität München, Building 56, Ingolstädter Landstraße 1, D-85764 Neuherberg, Germany.

E-mail: nicolas.beziere@helmholtz-muenchen.de

Published online Dec. 31, 2014.

COPYRIGHT (C 2015 by the Society of Nuclear Medicine and Molecular Imaging, Inc. sorption, this family of molecules can react with triplet oxygen to produce singlet oxygen, a highly reactive and cytotoxic oxidant (2). On the basis of photochemical reactions, photochemotherapy has been developed to selectively trigger tissue destruction in cancer (3) (4) or other diseases such as psoriasis (5). Since the arrival of Photofrin (porfimer sodium; Axcan Pharma) on the market in 1993 as the first Food and Drug Administration-approved synthetic photothermal therapy agent, this class of molecules has been expanded with formulations used for topical and systemic administration (6). Refining the core structure of phthalocyanines (notably into naphthalocyanines (7-9), close derivatives of Photofrin) and adapting delivery vehicles enabled fine tuning of photochemical and pharmacodynamic properties and solved potential solubility issues common in this chemical class (10).

Sensing of naphthalocyanines in vivo is important for understanding their biodistribution in tissues and for determining administered dose and illumination parameters, as underlined by fluorescence imaging studies $(6,11,12)$. However, classic fluorescence imaging methods cannot resolve depth accurately with good resolution and cannot offer a complete picture of biodistribution, especially considering the generally low quantum yield of naphthalocyanines.

Interestingly, this low quantum yield, combined with a high absorption cross-section, makes naphthalocyanines promising dyes for optoacoustic (photoacoustic) sensing. We hypothesized that optoacoustic imaging could offer an ideal modality to characterize naphthalocyanines in tissues because of the high-resolution, 3-dimensional imaging through several centimeters provided by optoacoustic methods. Optoacoustic imaging generates optical images by detecting the sound waves produced by photoabsorbers in response to their excitation with light of transient intensity $(13,14)$. Consequently, we investigated the characteristics of silicon 2,3-naphthalocyanine bi(trihexylsilyloxide) (SiNc, Fig. 1A) (15-17) in relation to its ability to generate optoacoustic signal. We were particularly interested in identifying the performance of SiNc over the more commonly used indocyanine green (ICG), an organic fluorophore with a high molecular extinction coefficient. ICG is a Food and Drug Administration-approved dye with high photoabsorbance but poor stability in vitro and in vivo and extremely rapid excretion (18-20). Finally, we uncovered how different pulsed laser light energy could trigger the $\mathrm{SiNc}$ reaction with oxygen and monitored the change in optical absorbance in tissuemimicking phantoms.

\section{MATERIALS AND METHODS}

Naphthalocyanine and ICG Solution Preparation

$\mathrm{SiNc}$ (Sigma-Aldrich) was prepared as an emulsion of $10 \%$ Cremophor EL, 1\% 1,2-propanediol, and 1\% dimethylformamide (16) 


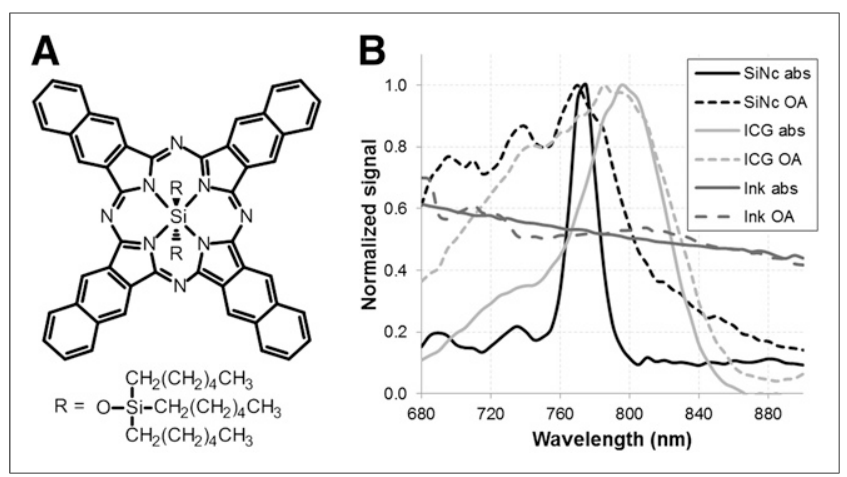

FIGURE 1. Structure of SiNc and its light absorption and optoacoustic spectra compared with ICG. (A) Structure of SiNc. (B) Overlay of normalized light absorption spectra (abs) and optoacoustic signal (OA) obtained in near-infrared wavelength range of naphthalocyanine emulsion (SiNc, black), ICG solution (light gray), and India ink (dark gray). Solutions were prepared with OD of 0.5 for ICG and SiNc and 0.3 for India ink.

(Sigma-Aldrich) in phosphate-buffered saline, $\mathrm{pH} 7.4$, by sonication. The concentration of the initial preparation was estimated using the absorbance of the solution and a molar extinction coefficient of $500,000 \mathrm{M}^{-1} \mathrm{~cm}^{-1}$ at around $40 \mu \mathrm{M}$. ICG solutions were prepared using the same emulsion. Reactive oxygen species (ROS) were measured using anthracene-9,10-dipropionic acid (ADPA, Sigma-Aldrich) as a scavenger. An initial solution containing $1.5 \cdot 10^{-3} \mathrm{~mol} \cdot \mathrm{L}^{-1}$ of ADPA was prepared and used at a $5 \%$ dilution in the SiNc solutions for the phantom experiments.

\section{Light Absorbance Measurements}

Absorbance of the solutions was recorded on an CUV-UV + USB2000 spectrometer (OceanOptics) and processed with SpectraSuite (OceanOptics), using a cuvette with a 1-cm light pathway and recording every $0.1 \mathrm{~nm}$. Absorbance of solutions containing ADPA was recorded in an ultraviolet-visible light plate interpreter (SpectraMax M2e; Molecular Device), using a 96-well plate and recording absorbance every $1 \mathrm{~nm}$.

\section{Optoacoustic System}

The experimental multispectral optoacoustic system was custom built and has been previously described extensively (21). Supplemental online materials (available at http://jnm.snmjournals.org) contain an extensive description of the system characteristics. The optoacoustic images were reconstructed using an interpolated matrix model inversion algorithm. Spectral unmixing was applied to detect signals from contrast agents within the samples $(22,23)$.

\section{Phantom Experiments}

Cylindric phantoms of 2-cm diameter were prepared using a gel made from distilled water, containing agar (Sigma-Aldrich) for jellification $(1.3 \% \mathrm{w} / \mathrm{w})$ and an intralipid $20 \%$ emulsion (SigmaAldrich) for light diffusion $(6 \% \mathrm{v} / \mathrm{v})$. The result was a gel with a reduced scattering coefficient of approximately $10 \mathrm{~cm}^{-1}$ and no specific absorbance so as to allow precise estimation of light energy deposition. A 3-mm-diameter cylindric inclusion containing the $\mathrm{SiNc}$ at an optical density (OD) of 0.5 was placed at the middle of the phantom, alongside a tube containing classic black India ink at an OD of 0.3 for intensity measurement references. The tube length was around $4 \mathrm{~cm}$, making for a total volume of solution of around $300 \mu \mathrm{L}$.

MSOT imaging was performed at a single position, approximately in the middle of the phantom. Data were acquired using 10 averages per wavelength and at every wavelength between $680 \mathrm{~nm}$ and $900 \mathrm{~nm}$ in steps of $5 \mathrm{~nm}$, resulting in a total acquisition time of around $1.5 \mathrm{~min}$. For photochemical experiments, a complete MSOT spectrum was recorded every $10 \mathrm{~min}$ for $1 \mathrm{~h}$, and pulsed light illumination was maintained between spectral acquisitions at $770 \mathrm{~nm}$ for $\mathrm{SiNc}$ or $800 \mathrm{~nm}$ for ICG. After the last time point, the solution was extracted from the MSOT phantom and its absorbance spectrum was recorded in the spectrophotometer described above. Every phantom experiment was performed in triplicate, and SD is indicated for the photochemical experiment.

\section{Animal Experiments}

Procedures involving animals were conducted in conformity with institutional guidelines and approved by the government of Upper Bavaria.

Three female CD-1 nu/nu mice (Charles River Laboratories) were inoculated with 1.5 million HT29 human colon cancer cells in $15 \mu \mathrm{L}$ of Matrigel (BD Biosciences) subcutaneously between the kidneys and the base of the tail, in the hip region. The cells were cultured in McCoy medium with $1 \%$ penicillin-streptomycin and $10 \%$ fetal calf serum in an atmosphere of $5 \% \mathrm{CO}_{2}$ at $37^{\circ} \mathrm{C}$.

For the imaging experiments, $200 \mu \mathrm{L}$ of an $\mathrm{SiNc}$ solution in a $25 \%$ Cremophor EL emulsion ( 2.5 OD) were injected intravenously in the tail vein after anesthesia using $2 \%$ isoflurane in oxygen. The anesthesia was maintained at $1.8 \%$ isoflurane in oxygen throughout the acquisition. Imaging was performed in the previously described MSOT system using transversal slices at the middle of the tumor, liver, and kidney regions at 680-, 700-, 730-, 750-, 770-, 800-, 830-, and 860-nm illumination wavelength for each position, using 50 averages per wavelength in order to minimize the influence of animal movement in the images. Image acquisition time for each slice was around $1 \mathrm{~min}$, and laser illumination was not maintained between acquisitions. The animals were sacrificed using cervical dislocation. The figures and calculations are based on a representative animal from the panel used, with all 3 animals displaying similar behavior.

\section{RESULTS}

\section{Spectral Measurements}

Figure 1 depicts the structure and absorption spectra of SiNc. Figure 1B shows the absorption spectra obtained from $\mathrm{SiNc}$ by optical and optoacoustic measurements from the same solution. Corresponding control measurements were obtained from black India ink and used for reference purposes, compensating for possible laser energy fluctuation during the optoacoustic measurements. As shown in Figure 1B, India ink absorbance and optoacoustic spectra correlated closely at all wavelengths. The optical and optoacoustic spectra of SiNc displayed good correlation above $770 \mathrm{~nm}$. However, in the lower wavelengths, the optoacoustic signal showed an elevated contribution compared with the optical measurements. Two absorption contributions at bands around 690 and $730 \mathrm{~nm}$ could nevertheless be seen on both the optical and the optoacoustic signal intensity curve. Spectrum measurements obtained from ICG exhibited a similar response (Fig. 1B). SiNc and ICG measurements were repeated multiple times at an $\mathrm{SiNc}$ concentration ranging from 0.1 to $1 \mathrm{OD}$. In all cases, the differences between SiNc spectra determined from the optical and optoacoustic signals were consistent. As a negative control, a Cremophor EL emulsion devoid of any photoabsorber was measured under similar conditions and did not display light absorption or a bias toward the optoacoustic signals compared with optical signals. The repeatability of optoacoustic spectra was also examined, to investigate the effects and overall stability of the illumination energy, and is shown in Figure 2A. 


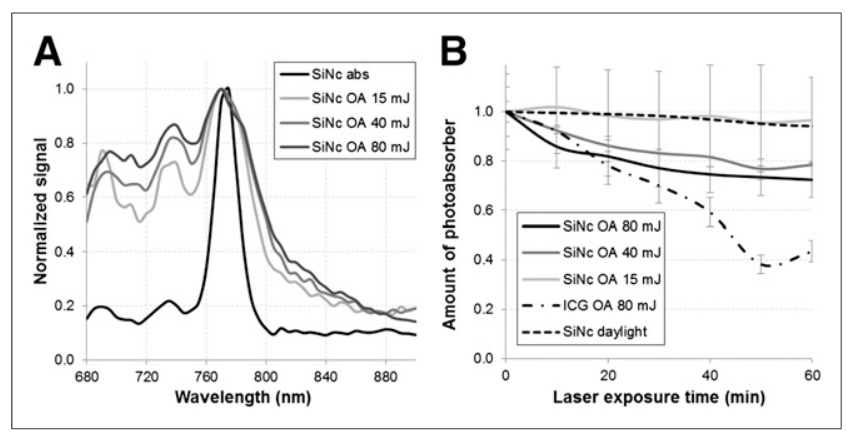

FIGURE 2. Influence of laser light on a solution of SiNc. (A) Normalized optoacoustic signal obtained in the near-infrared range for a SiNc solution using an average power of $15 \mathrm{~mJ}$ (optoacoustic signal [OA], $15 \mathrm{~mJ}$ ), $40 \mathrm{~mJ}(\mathrm{OA}, 40 \mathrm{~mJ})$, and $80 \mathrm{~mJ}(\mathrm{OA}, 80 \mathrm{~mJ})$ of laser energy per 10-ns pulse with the normalized light absorption spectrum (abs) of the same solution for reference. (B) Relative amount through time of $\mathrm{SiNc}$ detected in the optoacoustic system (OA) under constant laser pulse exposition at different energy steps (15, 40, and $80 \mathrm{~mJ})$, using light absorbance of similar solution left under daylight, as well as ICG decay under 80-mJ pulsed laser light as detected in optoacoustic system for comparison.

\section{SiNc Stability Measurements}

Because the reaction of naphthalocyanines with oxygen is lighttriggered, we investigated the behavior of a 0.5-OD SiNc emulsion in a light-scattering phantom under pulsed laser illumination in the optoacoustic imaging system. Figure $2 \mathrm{~B}$ shows the evolution of the optoacoustic signal of an SiNc emulsion illuminated by pulsed laser light at $770 \mathrm{~nm}$ at energies ranging between 15 and $80 \mathrm{~mJ}$ per pulse. At high energy settings (40 and $80 \mathrm{~mJ}$ ), loss of signal could be seen, resulting in around $70 \%$ of the initial value after $1 \mathrm{~h}$ of illumination. However, when only $15 \mathrm{~mJ}$ per pulse were used, no loss of optoacoustic signal was detected. At $80 \mathrm{~mJ}$, a similarly prepared ICG emulsion demonstrated faster photobleaching than SiNc, losing $60 \%$ of its initial optoacoustic signal after being illuminated for $1 \mathrm{~h}$ at $800 \mathrm{~nm}$. Transferring the emulsions into a spectrophotometer cuvette to obtain absorbance spectra after optoacoustic signal acquisition confirmed the findings, without any modification of the shape of the spectra. Interestingly, at the same absorbance values (0.5 OD), ICG proved around $60 \%$ more efficient to provide optoacoustic signal than $\mathrm{SiNc}$ at the initial time point (0.27 arbitrary units compared with 0.16 , respectively). However, given the increased stability of SiNc compared with ICG, its optoacoustic signal strength became comparable after $1 \mathrm{~h}$ of illumination.

\section{Detection of SiNc in Murine Subcutaneous Tumor Model}

To evaluate SiNc in vivo, we intravenously administered the dye emulsion to mice bearing a 1-cm-diameter HT29 tumor. Figure 3 shows MSOT images obtained from the tumor-bearing mouse 5, $15,30,45$, and 60 min after injection of SiNc. The distribution of SiNc was identified by spectral unmixing (24) applied to images obtained at multiple wavelengths. Figure $3 \mathrm{~A}$ represents the preinjection hemoglobin oxygenation map and depicts the anatomic features of a subcutaneous xenografted tumor. The main blood vessels feeding the tumor are indicated, as well as the tumor (Fig. 3A). Figures 3B-3F show an accumulation of the contrast agent in the core of the tumor and clearance of the molecule from the blood vessels surrounding the tumor. Because spectral unmixing allows for specific detection of $\mathrm{SiNc}$ on the basis of its absorption spectra, the data were further processed to reveal the biodistribution of SiNc after intravenous injection. Figure 4A depicts the optoacoustic spectra derived from SiNc in vivo in the tumor, overlaid with its optoacoustic spectra in a tissue-mimicking phantom. The in vitro and in vivo optoacoustic spectra derived from the phantom measurements and in vivo appear similar to each other but not identical. The differences might be attributed to the sparse spatial sampling applied in vivo and possibly spectral alterations due to the wavelength-dependent light fluence distribution (24). Similarly to the observations in Figure 2, Figure 4A also shows a discrepancy between the absorption spectrum measured by a spectrophotometer and the optoacoustic measurements. Although it is unclear why this discrepancy exists, it was consistent after the measurements were repeated multiple times and cannot be attributed to instrument calibration issues. Figure $4 \mathrm{~B}$ plots the change of SiNc optoacoustic signal strength detected in vivo as a function of time in the tumor region and in blood, from structures identified in Figure 3A, resulting in a plot of the pharmacokinetic profile of the molecule. Notably, the SiNc optoacoustic signal (Fig. 3B) peaked in the blood a few minutes after injection while being negligible in the tumor mass, indicating a successful intravenous injection. As confirmed by Figure 3C, SiNc showed a clear accumulation increase $15 \mathrm{~min}$ after injection, with a relative decrease in the blood compartment, pointing at accumulation of the compound in the tumor. The respective difference in SiNc optoacoustic signal strength between blood and tumor continued to grow until termination of the experiment $(1 \mathrm{~h}$, Fig. $3 \mathrm{~F})$. At the final time point of the acquisition, we could still observe the presence of the $\mathrm{SiNc}$ preparation in the liver and kidneys (Supplemental Fig. 1).

\section{Generation of ROS in Optoacoustic System}

Because pulsed laser illumination at the maximum absorbance wavelength induced an energy-dependent gradual loss of signal in phantoms (Fig. 2B), suggesting generation of ROS, we investigated the underlying physicochemical process of the apparent photobleaching. Figure 5 shows that the light absorbance of ADPA recorded in an optical spectrometer, after it was placed in solution with $\mathrm{SiNc}$ in a tissue-mimicking phantom, decreased after $1 \mathrm{~h}$ of illumination in the MSOT system at the maximum absorbance wavelength of SiNc $(770 \mathrm{~nm})$ with an energy of $40 \mathrm{~mJ}$ per 10-ns light pulse at a $10-\mathrm{Hz}$ repetition rate. Because the singlet oxygen reaction with ADPA causes loss of absorbance of this oxygen trap, this plot indicates that the chosen illumination settings are sufficient for triggering the reaction between $\mathrm{O}_{2}$ and $\mathrm{SiNc}$ and producing singlet oxygen. Comparatively, similar SiNc-ADPA solutions left for $1 \mathrm{~h}$ in the dark or under daylight did not show any significant decrease in ADPA absorbance, confirming the requirement for laser illumination.

\section{DISCUSSION}

We investigated the suitability of SiNc to generate optoacoustic signals and then used MSOT to monitor SiNc characteristics in vitro and in vivo. $\mathrm{SiNc}(16,17)$ is an interesting dye for optoacoustic studies because it displays sharp absorption in the near-infrared region (peak at $770 \mathrm{~nm}$ ), a spectral region where light can penetrate several centimeters within tissues. A sharp absorbance change is beneficial for the identification of photoabsorbers, since they can then be resolved by wavelength scanning and differentiated from the spectral signatures of others (25). In the particular case of SiNc, the absorbance change between 770 and $800 \mathrm{~nm}$ is remarkably steeper than with ICG, highlighting a beneficial feature of $\mathrm{SiNc}$ 


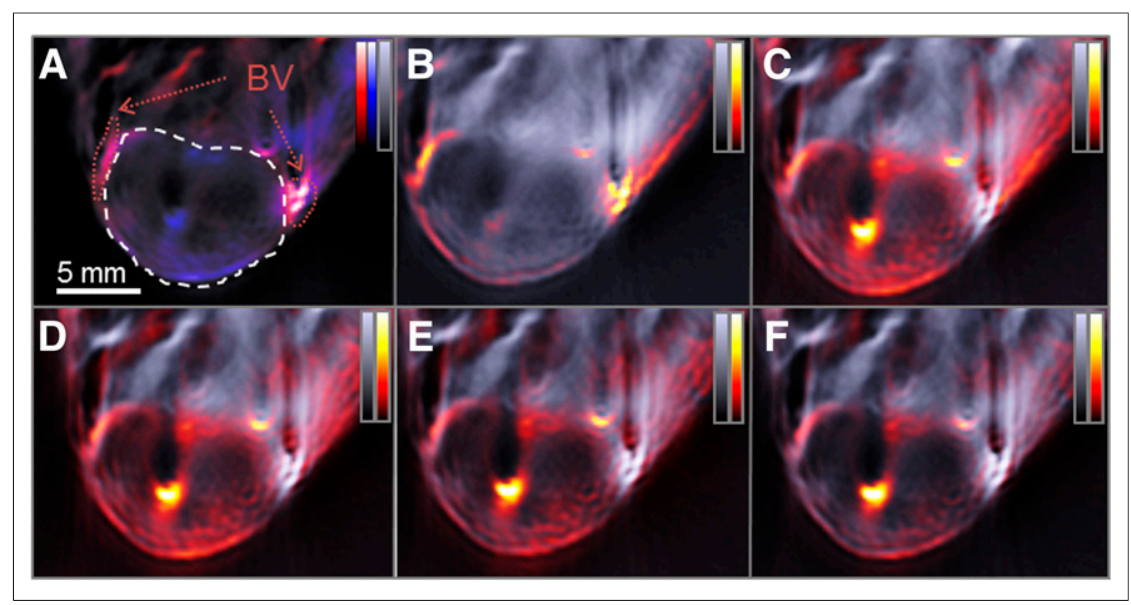

FIGURE 3. MSOT of a living mouse bearing a HT29 tumor on its back before and after intravascular injection of SiNc. Optoacoustic signal intensity obtained using 800-nm illumination wavelength (grayscale) is used as background in every frame and overlaid with the signal identified as coming from SiNc (hot scale). (A) Preinjection image, indicating tumor (dashed white circle) and surrounding blood vessels (BV, dotted red circles), overlaid with deoxygenated (blue scale) and oxygenated (red scale) hemoglobin signal. (B-F) Images acquired after 5 (B), 15 (C), 30 (D), $45(E)$, and $60(F)$ minutes after intravenous injection of SiNc in a tumor-bearing animal. $\pi-\pi^{*}$ transition resulting from the light absorption at $770 \mathrm{~nm}$ and leading to a lower apparent ultrasonic energy emitted and thus a flattened spectrum. Another possible explanation is a comparatively much greater influence of the small absorption peak located between 680 and $750 \mathrm{~nm}$, with higher-energy pressure waves being emitted. Several other physical parameters that could have an influence on the shape have been investigated, in particular the scanning direction (longer to shorter or shorter to longer wavelengths), SiNc concentration, and influence of Cremophor EL, without tangible results. Only when the amount of energy used was changed could we see a modification of the spectra, which confirms power-related mechanisms (Fig. 2A). Light energy was also found to affect the stability of the $\mathrm{SiNc}$, with the threshold for decomposition being between 15 and $40 \mathrm{~mJ}$ per pulse (Fig. 2B). Remarkably, even though the loss of signal due to light energy deposited on the sample was noticeable, it remained less marked than as a contrast agent for optoacoustic imaging. Measurements of the $\mathrm{SiNc}$ and ICG spectra showed a reproducible discrepancy between optical and optoacoustic observations in the 700- to 750-nm wavelength region (Fig. 1B). The spectra were acquired on the exact same solution, confirming that the $\mathrm{SiNc}$ was still in a monomeric state favored by the bulky axial silicon ligand, characterized by a sharp absorbance band at $770 \mathrm{~nm}$ (16).

This result was unexpected. Although methodologic errors are a first suspect, there is no justification for system bias in these studies. India ink, a stable photoabsorber presenting a characteristically flat absorbance spectra, was incorporated alongside the SiNc and ICG measurements to control for system bias but did not exhibit any discrepancy in shorter wavelengths, thus excluding system and experimental faults.

A possible explanation is the influence of the silicon ligand inside the complex, dampening the optoacoustic effect of the

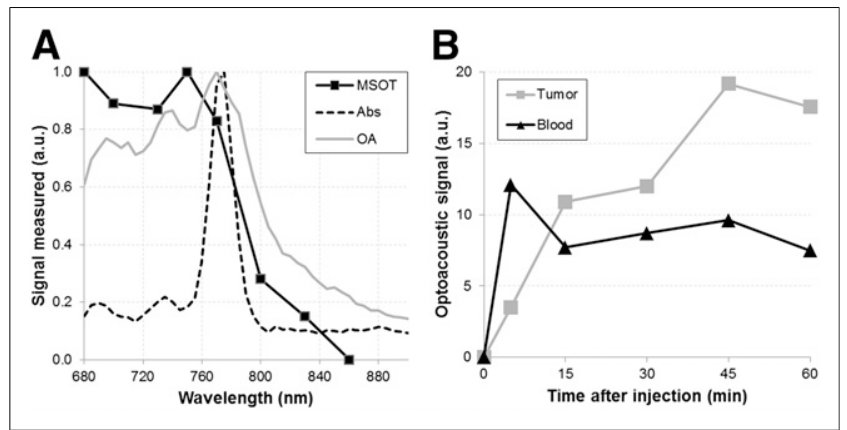

FIGURE 4. Spectral analysis of MSOT data acquired after injection of $\mathrm{SiNc}$ in a tumor-bearing mouse. (A) Light absorbance spectrum of naphthalocyanine solution (Abs) and optoacoustic signal strength obtained at different wavelength obtained in phantom (OA) and $1 \mathrm{~h}$ after intravascular injection in tumor-bearing mouse using principal component analysis of acquired MSOT data. (B) Maximum optoacoustic signal obtained from $\mathrm{SiNc}$ after identification of its spectrum using principal component analysis in blood compartment and tumor area after intravascular injection in HT29 tumor mouse model. a.u. = arbitrary unit.
ICG at comparable concentrations and thus justified the use of $\mathrm{SiNc}$ as a contrast agent able to provide a distinctive signature in the MSOT system with a 2.5 times higher molar extinction coefficient than ICG and less photobleaching $\left(500,000 \mathrm{M}^{-1} \mathrm{~cm}^{-1}\right.$ compared with $200,000 \mathrm{M}^{-1} \mathrm{~cm}^{-1}$, respectively). In terms of optoacoustic signal strength, even though ICG initially provides around $60 \%$ more signal than SiNc using the same OD for the solution, the difference quickly diminishes and becomes negligible after $1 \mathrm{~h}$, making SiNc almost twice as efficient as ICG for providing optoacoustic signal per mole. Thus, specific preparations of SiNc, such as liposomal or micellar, might overtake ICG as the organic contrast agent of choice for optoacoustic imaging, because providing more reliable contrast enhancement throughout the experiment in longitudinal studies is crucial, especially when comparing intensities between different time points.

Because the spectral characteristics of $\mathrm{SiNc}$ were proven interesting for optoacoustic contrast in vitro, identification of the compound in living animals was investigated after intravascular injection of an emulsion in a mouse bearing a subcutaneous tumor. Because emulsions of Cremophor EL are known to increase the circulation half-life of the formulated compounds, we monitored accumulation of the probe in the tumor and clearance from the blood flow. Interestingly, the signal coming from $\mathrm{SiNc}$ was still easy to identify even though the amount injected was low, and accumulation in the middle of the tumor, corresponding to a vascularized compartment, could be seen. Because blood vessels near the tumor were readily accessible, monitoring disappearance of the probe from the blood flow was also possible. Simultaneously, MSOT provided for real-time monitoring of SiNc accumulation in the tumor unavailable volumetrically by other means. The optoacoustic signal from the $\mathrm{SiNc}$ in vivo was similar to the one obtained in vitro, with an intense signal coming from the lower wavelength and a sharp drop between 770 and $800 \mathrm{~nm}$, making the SiNc signal easily identifiable.

Because the phthalocyanine-like complexes are known to display photodynamic reactions under high light energy, we hypothesized that this fact could at least partly explain the 


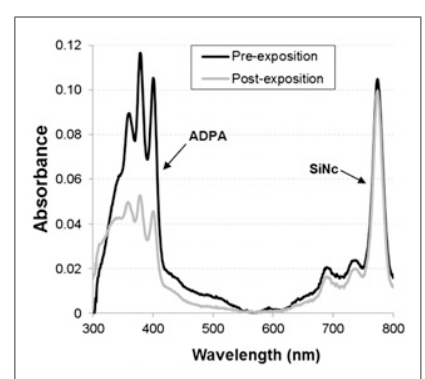

FIGURE 5. Evolution of the light absorption spectrum of an $\mathrm{SiNc}$ and ADPA solution under pulsed laser light. Ultraviolet-visible light absorption spectra of mixture of ADPA (maximum absorbance located around $350 \mathrm{~nm}$ ) and SiNc (absorption peak at $770 \mathrm{~nm}$ ) before (black line) and after (gray line) exposure to pulsed 770-nm laser light for 1 h using $40 \mathrm{~mJ}$ per pulse.

decrease in light absorbance of the solution after pulsed laser light exposition. The use of an ROS scavenger such as ADPA allows the phenomenon to be observed using visible absorbance measurements after reaction with ROS, a behavior that could be clearly seen under our experimental conditions (Fig. 5), suggesting that $40 \mathrm{~mJ}$ of energy per pulse was sufficient to trigger a photochemotherapy-like mechanism that we could not see under lowerenergy conditions. In our scattering phantom setup, $40 \mathrm{~mJ}$ of energy per pulse resulted in a total of approximately $100 \mathrm{~J} \cdot \mathrm{cm}^{-2}$ after $1 \mathrm{~h}$. These data should be compared with the classic way of generating ROS when applying a phthalocyanine derivative with a continuous-wave laser, typically using $100-400 \mathrm{~J} \cdot \mathrm{cm}^{-2}$ (16). However, comparison of pulsed and continuous laser energy deposition is not straightforward, and further experiments will be performed to provide quantitative data using pulsed laser energy. In vivo, and under our parameters, the light fluence condition renders estimation of the amount of light received by the tumor difficult; thus, it is currently not possible to anticipate whether continuous illumination during the experiment will prove sufficient to trigger the photochemotherapy effect on site.

SiNc proves to be a promising example of the phthalocyanine family for use as an MSOT imaging contrast agent. Its spectral properties, increased stability, and increased optoacoustic signal generation per mole, compared with ICG, should push it toward the foreground of this new imaging modality purely for contrast generation at even lower light energy settings. Additionally, when another property of phthalocyanines is considered, namely lighttriggered ROS generation, this family of polycyclic compounds exhibits promising applications in the new field of theranostics-in this particular case, a dual imaging-photochemotherapy approach. MSOT was able to monitor this photochemical reaction between $\mathrm{SiNc}$ and oxygen in vitro and provided access to the power-dependent generation of ROS in real time. Two possibilities for further development of naphthalocyanine exist for use in optoacoustics. First, by using non-ROS-generating derivatives, a new organic gold standard contrast agent might become accessible. Second, by specifically tailoring the naphthalocyanine to capitalize on energy-triggered ROS production, a new approach of theranostics with real-time monitoring is made available with direct applicability to various conditions, in particular for photochemical destruction of cancer cells. Naphthalocyanines and related structures have seen a large number of molecules synthesized (6), and a different screening approach based on optoacoustic-driven parameters could potentially benefit this new application field. It is anticipated that MSOT contrast agents will become a prominent field of research in the coming years and that the dual nature of the modality will be utilized to exhibit unique theranostic properties and applications.

\section{CONCLUSION}

As the position of optoacoustic imaging as a pivotal modality in modern imaging techniques is confirmed, the need to identify efficient contrast-generating agents becomes predominant. Classically used contrast agents such as ICG and gold nanorods have significant drawbacks such as instability and poor body tolerance. By investigating the potential of SiNc for optoacoustic imaging, we uncovered the significant benefits of the high molar extinction coefficient and sharp absorbance peak and the ease in identifying its distribution in vivo in a tumor model. Most notably, we showed that MSOT of SiNc was able to trigger the generation of ROS at the highest energy settings, effectively enabling photodynamic therapy. Additionally, MSOT was able to monitor the kinetics of this reaction by estimating the amount of naphthalocyanine present in the image. We believe this ability will open the window to real-time monitoring of the biodistribution of such an agent, while simultaneously enabling evaluation of its pharmacologic activity and efficiency, bringing MSOT firmly into the theranostic field.

\section{DISCLOSURE}

The costs of publication of this article were defrayed in part by the payment of page charges. Therefore, and solely to indicate this fact, this article is hereby marked "advertisement" in accordance with 18 USC section 1734. Financial support was provided by Deutsche Forschungsgemeinsaft (DFG), Sonderforschungsbereich-824 (SFB824), subproject A1 and the ERC Advanced Grant (233161) Next Generation in vivo imaging platform for postgenome biology and medicine MSOT. Vasilis Ntziachristos is an equity holder in iThera Medical GmbH. No other conflict of interest relevant to this article was reported.

\section{REFERENCES}

1. Roeder B, Naether D, Lewald T, Braune M, Nowak C, Freyer W. Photophysical properties and photodynamic activity in vivo of some tetrapyrroles. Biophys Chem. 1990;35:303-312.

2. Dougherty TJ, Gomer CJ, Jori G, et al. Photodynamic therapy. J Natl Cancer Inst. 1998;90:889-905.

3. Brown SB, Brown EA, Walker I. The present and future role of photodynamic therapy in cancer treatment. Lancet Oncol. 2004;5:497-508.

4. Davids LM, Kleemann B. Combating melanoma: the use of photodynamic therapy as a novel, adjuvant therapeutic tool. Cancer Treat Rev. 2011;37:465475 .

5. Tandon YK, Yang MF, Baron ED. Role of photodynamic therapy in psoriasis: a brief review. Photodermatol Photoimmunol Photomed. 2008;24:222230 .

6. Josefsen LB, Boyle RW. Photodynamic therapy and the development of metal-based photosensitisers. Met-Based Drugs. 2008;2008:276109276109.

7. Cuomo V, Jori G, Rihter B, Kenney ME, Rodgers MA. Liposome-delivered Si (IV)-naphthalocyanine as a photodynamic sensitiser for experimental tumours: pharmacokinetic and phototherapeutic studies. Br J Cancer. 1990;62: 966-970.

8. Margaron P, Langlois R, van Lier JE, Gaspard S. Photodynamic properties of naphthosulfobenzoporphyrazines, novel asymmetric, amphiphilic phthalocyanine derivatives. J Photochem Photobiol B. 1992;14:187-199.

9. Sessler JL, Magda DJ. Biomedical applications of texaphyrins [abstract]. Free Radic Biol Med. 2006;41(suppl):S8.

10. Wöhrle D, Shopova M, Muller S, Milev AD, Mantareva VN, Krastev KK. Liposome-delivered $\mathrm{Zn}$ (II)-2,3-naphthalocyanines as potential sensitizers for PDT: synthesis, photochemical, pharmacokinetic and phototherapeutic studies. J Photochem Photobiol B. 1993;21:155-165.

11. Lv F, He X, Wu L, Liu T. Lactose substituted zinc phthalocyanine: a near infrared fluorescence imaging probe for liver cancer targeting. Bioorg Med Chem Lett. 2013;23:1878-1882. 
12. Witjes MJ, Speelman OC, Nikkels PG, et al. In vivo fluorescence kinetics and localisation of aluminum phthalocyanine disulphonate in an autologous tumour model. Br J Cancer. 1996;73:573-580.

13. Ntziachristos V. Going deeper than microscopy: the optical imaging frontier in biology. Nat Methods. 2010;7:603-614.

14. Luke GP, Nam SY, Emelianov SY. Optical wavelength selection for improved spectroscopic photoacoustic imaging. Photoacoustics. 2013;1:36-42.

15. Wheeler BL, Nagasubramanian G, Bard AJ, Schechtman LA, Kenney ME. A silicon phthalocyanine and a silicon naphthalocyanine: synthesis, electrochemistry, and electrogenerated chemiluminescence. J Am Chem Soc. 1984;106:74047410 .

16. Brasseur N, Nguyen TL, Langlois R, et al. Synthesis and photodynamic activities of silicon 2,3-naphthalocyanine derivatives. J Med Chem. 1994;37:415-420.

17. Brasseur N, Ouellet R, Lewis K, Potter WR, van Lier JE. Photodynamic activities and skin photosensitivity of the bis(dimethylthexylsiloxy)silicon 2,3-naphthalocyanine in mice. Photochem Photobiol. 1995;62:10581065 .

18. Zhong J, Yang S, Zheng X, Zhou T, Xing D. In vivo photoacoustic therapy with cancer-targeted indocyanine green-containing nanoparticles. Nanomedicine (Lond). 2013;8:903-919.
19. Buehler A, Herzog E, Razansky D, Ntziachristos V. Video rate optoacoustic tomography of mouse kidney perfusion. Opt Lett. 2010;35:24752477.

20. Herzog E, Taruttis A, Beziere N, Lutich AA, Razansky D, Ntziachristos V. Optical imaging of cancer heterogeneity with multispectral optoacoustic tomography. Radiology. 2012;263:461-468.

21. Ma R, Taruttis A, Ntziachristos V, Razansky D. Multispectral optoacoustic tomography (MSOT) scanner for whole-body small animal imaging. Opt Express. 2009;17:21414-21426.

22. Buehler A, Rosenthal A, Jetzfellner T, Dima A, Razansky D, Ntziachristos V. Model-based optoacoustic inversions with incomplete projection data. Med Phys. 2011;38:1694-1704.

23. Rosenthal A, Razansky D, Ntziachristos V. Fast semi-analytical model-based acoustic inversion for quantitative optoacoustic tomography. IEEE Trans Med Imaging. 2010;29:1275-1285.

24. Tzoumas S, Deliolanis NC, Morscher S, Ntziachristos V. Unmixing molecular agents from absorbing tissue in multispectral optoacoustic tomography. IEEE Trans Med Imaging. 2014;33:48-60.

25. Ntziachristos V, Razansky D. Molecular imaging by means of multispectral optoacoustic tomography (MSOT). Chem Rev. 2010;110:2783-2794. 